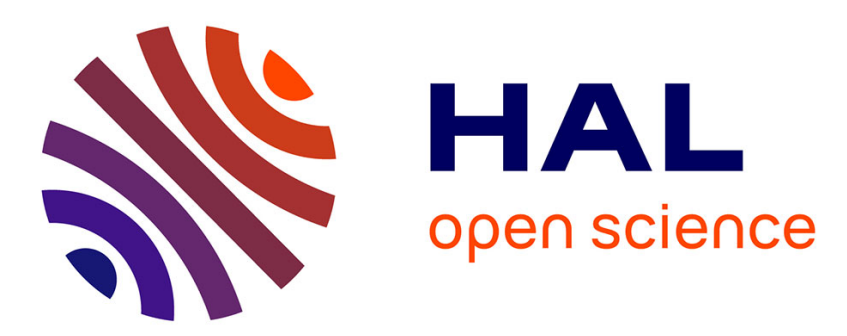

\title{
Why is Frequency Channel Diversity so Beneficial in Wireless Sensor Networks?
}

Liviu-Octavian Varga, Martin Heusse, Roberto Guizzetti, Andrzej Duda

\section{To cite this version:}

Liviu-Octavian Varga, Martin Heusse, Roberto Guizzetti, Andrzej Duda. Why is Frequency Channel Diversity so Beneficial in Wireless Sensor Networks?. IFIP Wireless Days, IFIP, Mar 2016, Toulouse, France. hal-01287518

\author{
HAL Id: hal-01287518 \\ https://hal.science/hal-01287518
}

Submitted on 14 Mar 2016

HAL is a multi-disciplinary open access archive for the deposit and dissemination of scientific research documents, whether they are published or not. The documents may come from teaching and research institutions in France or abroad, or from public or private research centers.
L'archive ouverte pluridisciplinaire HAL, est destinée au dépôt et à la diffusion de documents scientifiques de niveau recherche, publiés ou non, émanant des établissements d'enseignement et de recherche français ou étrangers, des laboratoires publics ou privés. 


\title{
Why is Frequency Channel Diversity so Beneficial in Wireless Sensor Networks?
}

\author{
Liviu-Octavian Varga*ף, Martin Heusse*, Roberto Guizzetti $₫$, and Andrzej Duda*. \\ ${ }^{*}$ Grenoble Alps University, Grenoble Institute of Technology, CNRS Grenoble Informatics Laboratory, Grenoble, France. \\ ISTMicroelectronics, Crolles, France \\ Email: \{firstname.lastname\}@imag.fr, \{firstname.lastname\}@st.com
}

\begin{abstract}
In dense wireless sensor networks, a multichannel MAC is a good means to reduce channel contention and increase frame reception probability. In this paper, we report on experiments with transmissions on various channels in the $2.4 \mathrm{GHz}$ ISM band and find more channel diversity than expected: this effect is particularly exacerbated at a short range, but it also has a significant impact at any distance. Moreover, we find that wireless sensor nodes have a radiation pattern that changes significantly with the frequency channel. This feature is inherent to the size of the sensor node, in which the antenna necessarily interferes with other components. The first consequence of this finding is that frequency diversity in sensor networks is even more effective than generally thought, and conversely, single channel communication schemes should be avoided as long as the power budget is not very comfortable.
\end{abstract}

Keywords-802.15.4, coherence band, channel hopping, radio propagation

\section{INTRODUCTION}

In the context of a growing number of connected devices forming potentially dense wireless networks, using the whole available spectrum becomes unavoidable. IEEE 802.15.4 defines 16 channels in the $2.4 \mathrm{GHz}$ band with the width of $2 \mathrm{MHz}$ and $5 \mathrm{MHz}$ interchannel spacing. Besides segregating the transmissions, changing the channel allows to benefit from the diversity due to the sensitivity of multipath fading to the central frequency. In other words, there can be a channel between two nodes that works well, but during the same time frame, this channel gives very bad performance with other nodes. For all these reasons, the IEEE 802.15.4e-2012 amendment [1] specified channel hopping protocols such as TSCH (Time Synchronized Channel Hopping) or DSME (Deterministic and Synchronous Multi-channel Extension).

Even though a lot of research aims at harnessing channel diversity, most of the studies invoke multipath fading as the reason of bad communication on certain channels between two given nodes. Two solutions exist to improve performance: either change the position of a node or the antenna, or change the frequency. The first solution is inapplicable in most cases because access to the node might not always be possible. Taking advantage of antenna diversity is also challenging to implement for cost and space constraints.

Fortunately, even for short range and line of sight communications, our experiments show that there is a lot of diversity when changing channels, more than multipath fading itself can explain. We posit that the entire wireless sensor board has a role in radio propagation. On certain frequencies and in many directions, its presence either attenuates the radio signal or amplifies it. This effect is unavoidable for compact nodes and it has a noticeable impact within the band of interest.

We start the paper with a simple radio propagation model and present selected real world measurements of commu- nications involving GreenNet nodes (Fig. 1) developed by STMicroelectronics [2]. The board is based on a System on Chip STM32L low power microcontroller with a test-chip RF200 radio and a ceramic RainSun antenna [3].
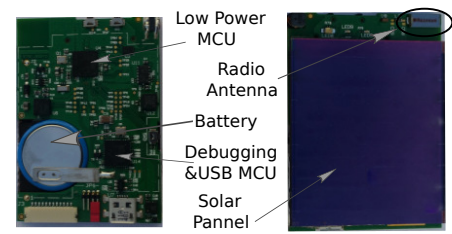

Figure 1: GreenNet board

\section{CHANNEL DIVERSITY OF 802.15.4 IN THE 2.4GHz ISM BAND}

In presence of two propagation paths, the coherence band $B_{c}$ is the frequency shift for which the phase difference at the receiver changes by $180^{\circ}$. It is a function solely of $\delta_{d}$, the path length difference between the two propagation paths:

$$
B_{c}=\frac{C}{2 \cdot \delta_{d}}
$$

[4]. This expression is only relevant for a pair of paths with similar incident power at the receiver: in fact, if the difference is of the same order of magnitude as the shortest path length, then free space attenuation lessens the impact of the secondary path.

Consequently, the coherence band for short distances is inherently larger than the band for longer distances, where larger $\delta_{d}$ corresponds to similar received powers on the considered paths. For instance, with $1 \mathrm{~m}$ between the sender and the receiver, a reflection on a wall $1 \mathrm{~m}$ behind the receiver reaches the receiving antenna with a power at most one $9^{\text {th }}$ of the power on the direct path, so it will not hurt much the direct path wave. Conversely, at e.g. $5 \mathrm{~m}$ or more, the reflected signal may mostly cancel out the direct wave [5]. When the sender and the receiver are further and further apart, the coherence band eventually shrinks well below $0.1 \mathrm{MHz}$ at distances of the order of $1 \mathrm{~km} \mathrm{[6].}$

For $\delta_{d}=1 \mathrm{~m}$ (think of a reflection on a wall $50 \mathrm{~cm}$ behind the sender or the receiver) the coherence band is $150 \mathrm{MHz}$, or 30 times the 802.15 .4 channel spacing $(5 \mathrm{MHz})$, whereas the ISM band is "only" $80 \mathrm{MHz}$ wide. In this case, the whole range of the available transmission frequencies is too small to exploit the diversity from varying multipath recombinations. From this simple analysis, it appears unlikely that a frequency shift by $5 \mathrm{MHz}$ brings any change due to multipath fading at a short distance. The next section considers a more complex synthetic model that captures with more accuracy how the combination of multiple radio waves changes with the channel center frequency. 


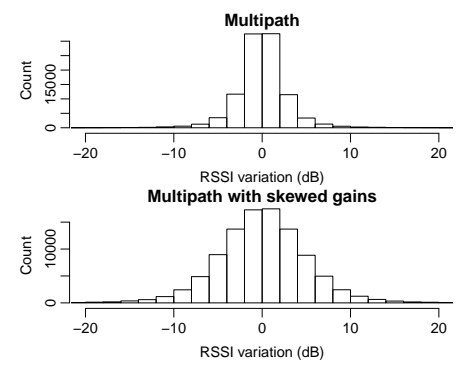

(a)

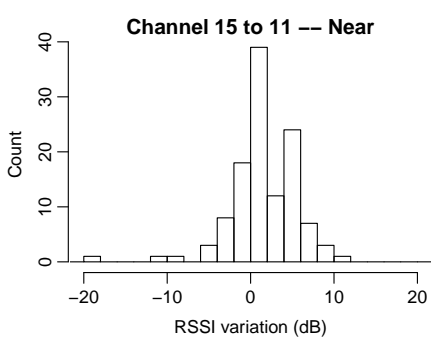

(b)
Figure 2: Fig. 2a: Computed RSSI variations at $1 \mathrm{~m}$ with one dominant path, when changing the frequency by $20 \mathrm{MHz}$ (4 channels or a $0.8 \%$ wavelength variation). Top: variations solely due to the phase changes at the receiver; bottom: with additional random gain changes among the paths; Fig. 2b: Experimentally measured RSSI variations at $1 \mathrm{~m}$

\section{A. Considering more than two propagation paths}

With more paths, the recombination at the receiver is harder to predict but, in general, it is expected that the coherence band shrinks. To assess the influence of a channel change, we consider a simple model with one direct (dominant) path and several scatterers or reflectors (for a total of 8 waves, in this section) placed at random positions from the immediate vicinity of the sender or the receiver up to $600 \%$ of the direct path length. Each path receives varying amounts of power, and all paths except the direct one, are attenuated by $3 \mathrm{~dB}$, which is rather typical for a reflected wave [7]. The histograms of the gain variations for a direct path of $1 \mathrm{~m}$ appear in Figure 2a, respectively, for a frequency shift of 4 channel spacing gaps $(20 \mathrm{MHz})$. Multipath propagation is only modeled for the top histogram, whereas random gain variations of up to $5 \mathrm{~dB}$ at the sender and the receiver are applied for the bottom histogram.

At $1 \mathrm{~m}$, much like previously experienced at a few meters in the $433 \mathrm{MHz}$ band [8], we do not expect that changing frequency would have much effect: accordingly, there is hardly any gain variation above $6 \mathrm{~dB}$ in the histogram at the top in Figure $2 \mathrm{a}$. However, Figure $2 \mathrm{~b}$ shows a different behavior: at $1 \mathrm{~m}$ indoors, gain changes of $6 \mathrm{~dB}$ or above are common. So, there is something more: in the model, we need to randomly change the channel gains over the various paths by e.g. $5 \mathrm{~dB}$ at the sender and the receiver when shifting the channel frequency to get gain variations reaching this magnitude, in the histogram at the bottom of Figure 2a. This last gain distribution significantly differs from the measurements presented in Figure 2b: in the real world conditions, a frequency change does not change the gains randomly, but there is a general improvement when going from channel 15 to 11, with some exceptions.

Our point is that multipath fading is insufficient by itself to explain these gain variations when changing channels. In the next section, we consider the case with a single path to get insight into another possible cause for the gain variations.

\section{OUTDOOR MEASUREMENTS: RADIATION PATTERNS $v s$. THE TRANSMISSION FREQUENCY}

The experiment takes place at a large empty parking lot with the receiver node in the middle, one meter above the ground and the sender on a robot that circles around the receiver. The constant distance between the two nodes is maintained using a string between the center of the circle and

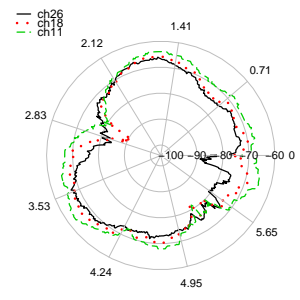

(a) receiver placed flat (horizontal antenna)
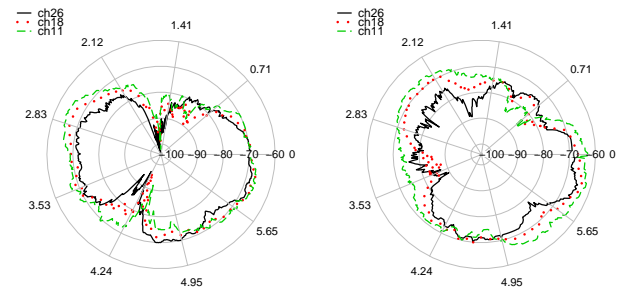

(b) receiver placed vertically(c) receiver placed horizon(horizontal antenna) tally

(vertical antenna)
Figure 3: Reception patterns for a GreenNet board

the robot. Each experiment corresponds to a different position of the sender (flat, vertical pointing up and then, laterally).

Fig. 3 shows the average RSSI (Received Signal Strength Indicator) for 20 packets/channel at each robot position. We can observe that the signal strength notably varies with the aspect angle. The pattern in Figure $3 \mathrm{~b}$ is expected-it corresponds to a typical dipole radiation pattern. What is less expected, though, is the difference of up to $10 \mathrm{~dB}$ from one channel to another in many directions. Furthermore, channel 11 has a higher signal reception strength in many, but not all, directions, which matches the bias already noticed at a short range in Figure $2 b$.

\section{A. Origin of the phenomenon}

We consider a board that integrates the antenna, the circuit board, and a solar panel. So, the transmitted radio signal is in fact the combination of the antenna radiation with the radio waves scattered and reflected by the other components, which is unavoidable. Even with a single path in the environment, the receiver gets a combination of waves from the transmitter, and actually creates itself a set of secondary radio waves. It turns out that the combination of scattered waves is sensitive to the transmission frequency at the granularity of the 802.15.4 channel spacing.

If this effect is better understood, and taking for granted that advanced sensor network MAC layer will include some form of channel agility, one can imagine designing a sensor board in such a way that the difference from one channel to the next is exacerbated, to increase the diversity.

\section{INDOOR MEASUREMENTS}

In this experiment, the sender is static and the receiver is attached to a small robot. The sender sends three groups of packets on different channels $(11,18,26)$ and the receiver sends back statistics. Then, the sender commands the robot to move forward etc. In the first part of the experiment, nodes are in line of sight, whereas after four meters, they are not in the direct view any more, as the robot moves out of the room. In each position, the sender sends 20 packets on each of the three channels (so 60 packets/position) and waits for the receiver to send back the RSSI measured for each received packet. The emission power is set to $0 \mathrm{dbm}$ and there is no retransmission when the hardware acknowledgement is not received.

Figure 4a shows the RSSI measured on each channel over the $10 \mathrm{~m}$ course of the robot. The RSSI follows a general trend: it goes down as the robot moves away. Nevertheless, 

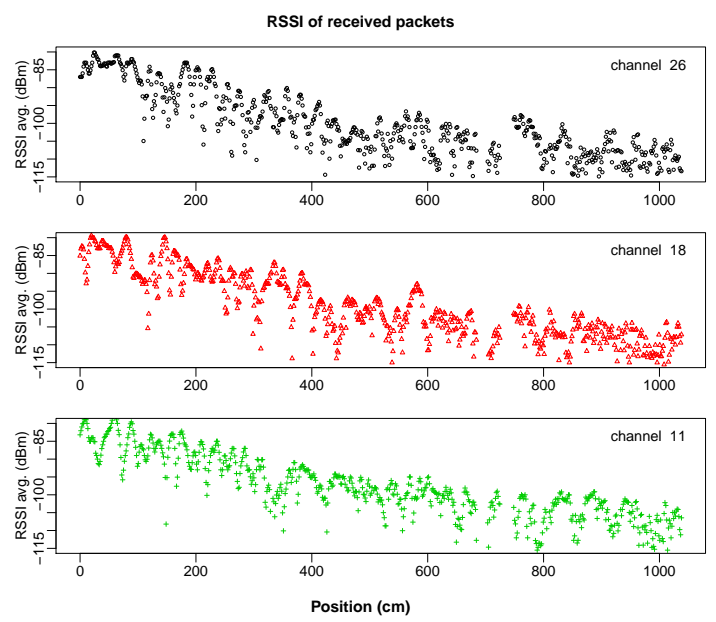

(a) RSSI value for each position for channels 11, 18, 26

Figure 4: Indoor evaluation

locally, the RSSI varies considerably even when the sender and the receiver are in the same room, and clearly differs from one channel to another: signal fadings on each channel are rather uncorrelated although the measurements were taken at very near instants. Obviously, in the same room, RSSI is high enough to warrant successful reception, whereas it gets more challenging at the other end of the corridor.

\section{RELATED WORK}

Watteyne et al. [4] considers that deep fading caused by destructive interference can be overcome by switching the frequency or the location of a node. They estimate that the coherence band for $2.4 \mathrm{GHz}$ varies from $5 \mathrm{MHz}$ for long ranges up to $15 \mathrm{MHz}$ for extremely short links, and we suspect that this finding is also due to the physical configuration of the sender and receiver used in experiments.

In the experiments made by Varela and Sánchez [9], they find that the $90 \%$ coherence band is of the order of 5 to $10 \mathrm{MHz}$ at $10 \mathrm{~m}$, and $10 \mathrm{MHz}$ or above at $3 \mathrm{~m}$. This coherence band corresponds to $B_{0.9}=\frac{1}{50 \sigma_{\tau}}$, where $\sigma_{\tau}$ is the mean delay among the paths weighted by the received power, which results in $50 \mathrm{MHz}$ and more for the $50 \%$ coherence band $(3 \mathrm{~dB}$ variation) $\left(B_{0.5}=\frac{1}{5 \sigma_{\tau}}\right)$. This value is much larger than what we observe with our nodes. Besides, they also set out that the coherence band is generally larger for the copolar component than for crosspolar one whereas in most other studies, only the copolar antenna positions are considered. This is not surprising as the crosspolar setup reduces the gain on the direct path.

Wysocki and Zepernick [10] presented a characterization of the indoor radio propagation in the $2.4 \mathrm{GHz}$ band with measurements of fading characteristics, multipath parameters, and background interference. In a room of $10 \mathrm{~m}$ by $8 \mathrm{~m}$, they find lower coherence bands than Varela, which may be due to an environment that contains many scatterers.

Giorgetti et al. [11] proposed an approach based on directional antennas placed on different sides of a node, using specific 802.15.4 antennas. This solution can be used to overcome the imperfection of a board with a ceramic antenna, but the main drawback is the size of the device-GreenNet nodes are only $4 \mathrm{~mm}$ thick.

\section{CONCLUSIONS}

In this paper, we have provided an experimental evidence of the fact that changing channels can have a significant influence on the radio propagation and consequently, on the quality of the link between two or more devices. However, in the case of close range communications in line of sight, channel diversity is not only due to multipath, but also to other phenomena. It is hard to believe that just multipath fading can have an impact of up to $10 \mathrm{~dB}$ when changing channels in line of sight at a short distance. We postulate that the difference most probably comes from the board construction. As the antenna interferes with the board, its ground plane and, in our case, the solar panel, it strongly affects the radiation pattern and renders it sensitive to the transmission frequency.

This fact may become an issue for the basic 802.15.4 scheme that do not support channel hopping. In this case, nodes need to be laid out with a comfortable range margin to compensate for the channel gain uncertainty. Even with stable omnidirectional antennas and a copolar placement of nodes, multipath fading is unavoidable, and may only be overcome with antenna diversity, which in turn imposes a much larger footprint. On the contrary, 802.15.4 channel hopping schemes may significantly benefit from the gain variability with respect to the transmission channel, so we can actually design boards to increase the level of diversity.

\section{REFERENCES}

[1] 802.15.4e-2012: IEEE Standard for Local and metropolitan area networks-Part 15.4: Low-Rate Wireless Personal Area Networks (LRWPANs) Amendment 1: MAC sublayer, IEEE Std., 16 April 2012.

[2] L.-O. Varga, G. Romaniello, M. Vucinic, M. Favre, A. Banciu, R. Guizzetti, C. Planat, P. Urard, M. Heusse, F. Rousseau, O. Alphand, E. Duble, and A. Duda, "Greennet: An energy-harvesting ip-enabled wireless sensor network," Internet of Things Journal, IEEE, vol. 2, no. 5, pp. 412-426, Oct 2015.

[3] “RainSun." [Online]. Available: http://www.rainsun.com

[4] T. Watteyne, S. Lanzisera, A. Mehta, and K. Pister, "Mitigating Multipath Fading through Channel Hopping in Wireless Sensor Networks," in Communications (ICC), 2010 IEEE International Conference on, May 2010, pp. 1-5.

[5] D. Sexton, M. Mahony, M. Lapinski, and J. Werb, "Radio channel quality in industrial wireless sensor networks," in Sensors for Industry Conference, 2005, Feb 2005, pp. 88-94.

[6] D. C. Cox and R. P. Leck, "Correlation Bandwidth and Delay Spread Multipath Propagation Statistics for 910-MHz Urban Mobile Radio Channels," Communications, IEEE Transactions on, vol. 23, no. 11, pp. 1271-1280, 1975.

[7] O. Landron, M. Feuerstein, and T. Rappaport, "A comparison of theoretical and empirical reflection coefficients for typical exterior wall surfaces in a mobile radio environment," Antennas and Propagation, IEEE Transactions on, vol. 44, no. 3, pp. 341-351, Mar 1996.

[8] P. Tuset-Peiró, A. Anglès-Vazquez, J. López-Vicario, and X. VilajosanaGuillén, "On the suitability of the $433 \mathrm{mhz}$ band for $\mathrm{m} 2 \mathrm{~m}$ low-power wireless communications: propagation aspects," Transactions on Emerging Telecommunications Technologies, vol. 25, no. 12, pp. 1154 1168, 2014. [Online]. Available: http://dx.doi.org/10.1002/ett.2672

[9] M. S. Varela and M. G. Sánchez, "RMS Delay and Coherence Bandwidth Measurements in Indoor Radio Channels in the UHF Band," Vehicular Technology, IEEE Transactions on, vol. 50, no. 2, pp. 515525, 2001.

[10] T. A. Wysocki and H.-J. Zepernick, "Characterization of the Indoor Radio Propagation Channel at $2.4 \mathrm{GHz}$," Journal of telecommunications and information technology, pp. 84-90, 2000.

[11] G. Giorgetti, A. Cidronali, S. K. Gupta, and G. Manes, "Exploiting LowCost Directional Antennas in 2.4 GHz IEEE 802.15.4 Wireless Sensor Networks," in Wireless Technologies, 2007 European Conference on. IEEE, 2007, pp. 217-220. 